\title{
AMPT partially reverses euphoric but not psychomotor effects of alcohol in humans
}

\author{
THOMAS H. RAMMSAYER \\ University of Giessen, Giessen, Germany \\ and \\ WOLFGANG H. VOGEL \\ Thomas Jefferson University, Philadelphia, Pennsylvania
}

\begin{abstract}
There are two dopaminergic brain systems that may be involved in the action of alcohol-the mesolimbic dopamine (DA) system, presumably by mediating alcohol-induced euphoria, and the mesostriatal DA system, by acting as a tonic regulatory system. In a double-blind placebo-controlled experiment, either $1,750 \mathrm{mg}$ of alpha-methyl-para-tyrosine (AMPT; an inhibitor of tyrosine hydroxylase), $0.65 \mathrm{~g} / \mathrm{kg}$ of alcohol, both substances combined, or a placebo only were applied to 60 healthy male subjects. Pretreatment with AMPT resulted in a pronounced reduction of urinary levels of DA, DOPAC, and HVA and caused a marked reduction in alcohol-induced euphoria, as indicated by self-ratings of joyfulness. Alcohol caused a decrease in experienced feelings of alertness and concentration as well as in critical-flicker-fusion (CFF) threshold values. In addition, lift-off time, representing a subcomponent of reaction time, was increased by alcohol, but was unaffected by AMPT. This pattern of results supports the notion of two distinct dopaminergic brain systems mediating behavioral effects of alcohol and, further, is in agreement with the hypothesis of differential sensitivity of dopaminergic and GABAergic mechanisms underlying the biphasic action of alcohol.
\end{abstract}

The ability of drugs such as amphetamine, cocaine, morphine, heroin, and alcohol to produce euphoric sensations and, thus, a rewarding experience appears to be the crucial factor in the development of psychological drug dependence or addiction (Engel \& Carlsson, 1977). This means that drugs of abuse are taken because of their ability to pharmacologically activate brain systems involved in primary reinforcement (Bozarth, 1987). As a common characteristic of such drugs, there seems to exist a relationship between their central stimulatory effects and their effects on the mesolimbic dopamine (DA) system (Fibiger \& Phillips, 1987). Mesolimbic dopaminergic neurons, projecting from the ventral tegmental area (VTA) to the nucleus accumbens, have been identified as the neurobiological substrate most likely responsible for these stimulatory and euphoric actions (Bozarth \& Wise, 1981; Fibiger \& Phillips, 1986, 1987; Gallistel, Shizgal, \& Yeomans, 1981; Khatib, Murphy, \& McBride, 1988; Wise, 1983, 1988). One of the most widely studied groups of addictive substances consists of the amphetamines. Amphetamines enhance the release of DA and, concomitantly, reduce its reuptake and inactivation (Wise, 1988). These actions lead to an increase in the concentrations of synaptic DA and to an enhancement of the activity of the mesolimbic DA system, result-

This work was supported by Grant Ra 450/1-1 from the German Research Foundation to T.H.R. and by Grant AA06017 from the Public Health Service to W.H.V. Correspondence should be addressed to T. H. Rammsayer, Department of Psychology, University of Giessen, OttoBehaghel-Strasse. 10F, D-35394 Giessen, Germany (e-mail: thomas. rammsayer@psychol.uni-giessen.de). ing in the euphoric effects of the amphetamines. This may also be true for alcohol (Engel \& Carlsson, 1977), although alcohol has a variety of additional effects as well, such as on cell membranes, on certain enzymes, on the sodiumdependent reuptake of calcium, and on various neurotransmitter systems (Dial, 1992; Lee \& Becker, 1989).

Pharmacological studies have shown that the euphoric effects of amphetamines or alcohol can be markedly reduced if the synthesis of brain DA is pharmacologically inhibited, leading to a DA deficiency in dopaminergic neurons and a decrease in the concentration of synaptic DA. In animal studies, alpha-methyl-para-tyrosine (AMPT), which effectively blocks the biosynthesis of catecholamines by inhibiting the hydroxylation of tyrosine to L-dopa, was found to antagonize the typical behavioral manifestions of amphetamine and alcohol exposure (Davis \& Smith, 1973; Engel, Strömbom, Svensson, \& Waldeck, 1974). Also, in human subjects, AMPT caused a dose-dependent decrease in amphetamine-induced euphoria in chronic amphetamine users (Jönsson, Änggård, \& Gunne, 1971; Jönsson, Gunne, \& Änggård, 1969). In addition, the results of two other studies (Ahlenius, Carlsson, Engel, Svensson, \& Södersten, 1973; Engel \& Carlsson, 1977) suggest that pretreatment with AMPT is also effective in reducing alcohol-induced stimulation and euphoria in humans. Both studies found a significant reduction of alcohol-induced euphoria after AMPT pretreatment as compared with placebo administration. Unfortunately, due to inadequate experimental designs, the outcome of neither of these studies can unequivocally support the hypothesis that inhibition of DA synthesis by AMPT reduces alcohol- 
induced euphoria. Therefore, one goal of the present study was to provide better evidence that alcohol-induced euphoria can indeed be reduced by AMPT-induced inhibition of DA synthesis.

In addition to the mesolimbic DA system, there is another important DA system, that projects mainly from the substantia nigra to the striatum and that, therefore, is referred to as the mesostriatal DA system. On the basis of animal experiments, Stricker and Zigmond (1986; Heffner, Zigmond, \& Stricker, 1977; Zigmond, Heffner, \& Stricker, 1980) have proposed a behavioral model for the action of the mesostriatal DA system. According to this model, dopaminergic neurons reduce the threshold for behavioral responses to sensory input. In this view, the mesostriatal DA system acts as a tonic regulatory system whose function is thought to be as follows: Each sensory input triggers two processes-a specific process that stimulates certain neuronal pathways necessary for eliciting an appropriate behavioral response, and a nonspecific process that is responsible for a generally activating action of sensory input (see Figure 1). The nonspecific process consists of three functional steps: First, every sensory input stimulates the mesostriatal DA neurons; second, an inhibitory control mechanism is modulated by the activity of the mesostriatal DA neurons; and, finally, this inhibitory control mechanism, probably characterized by GABA neurons (Björklund \& Lindvall, 1986), exerts inhibitory influence on the neuronal pathways that are responsible for the specific process. This inhibitory influence is modulated by the mesolimbic DA system, in that an increase in its activity leads to a reduction of the inhibition by the GABAergic neurons, while a decrease in its activity leads to an enhancement of the inhibition by these neurons. Thus, the specific process is modulated by the mesostriatal DA system via the inhibitory control mechanism. Consequently, any decrease in mesostriatal dopaminergic activity below a critical threshold will cause behavioral suppression due to the fact that sensory input fails to elicit an appropriate behavioral response.

At the behavioral level, the influence of the nonspecific process on the transformation of a sensory input into proper motor output is, for instance, reflected by changes in reaction time (Rammsayer, Netter, \& Vogel, 1993). In

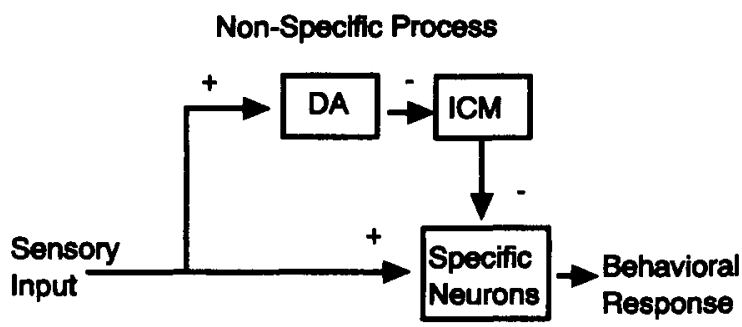

\section{Specffic Process}

Figure 1. Model relating mesostriatal dopaminergic activity to behavioral response (modified after Björklund \& Lindvall, 1986). DA, mesostriatal dopamine system; ICM, inhibitory control mechanism; +, facilitation; - , inhibition. this respect, relatively short reaction times can be expected in case of an optimal dopaminergic modulation. This means that a sensory input is efficiently processed and causes a fast and adequate response. On the other hand, an increase in the activity of the inhibitory control mechanism due to insufficient dopaminergic modulation should result in increase of reaction time. On the basis of these considerations, a second goal of the present study was to investigate the influence of alcohol and AMPT on dopaminergic modulation of behavioral responsiveness by applying a reaction time paradigm.

\section{METHOD}

\section{Subjects}

In a double-blind placebo-controlled study, either $1,750 \mathrm{mg}$ of AMPT, $0.65 \mathrm{~g} / \mathrm{kg}$ of ethanol, both substances combined, or two respective placebos were administered to 60 healthy male subjects ranging from 20 to 35 years of age (mean age $=24.4$ years). The subjects were randomly assigned to one of the four experimental conditions consisting of 15 subjects each. Further, the subjects of all experimental groups were matched for trait anxiety (using the trait anxiety scale; Spielberger, Gorsuch, \& Lushene, 1970) and drinking habits (using the questionnaire on alcohol consumption; Netter \& Vogel, unpublished). Only subjects who were either nondrinkers or moderate drinkers (ethanol intake less than $45 \mathrm{~g} /$ week) were enrolled in this study. In addition, the subjects were selected according to the following inclusion criteria: they had to be nonsmokers, with no history of chronic drug intake, no past or present psychotherapy or psychiatric treatment, no allergies, no chronic endocrine or cardiovascular disease, and no signs of present acute or chronic infections or gastrointestinal diseases. All subjects were informed about the study protocol and the drugs used in this experiment, and gave their written informed consent. For taking part in this study, they were paid $\$ 40$.

\section{Choice Reaction Time}

Choice reaction time was measured by a ZAK Leeds Psychomotor Tester. The apparatus comprises an array of six lights arranged in a semicircle. When one of these lights was turned on, the time required to lift the hand from a start button was scored as lift-off time, and the time required to move the finger to the corresponding response button located below each light was measured separately as movement time. Stimulus presentation was randomly generated. Thirty presentations were conducted and mean times for lift-off and movement were computed.

\section{Critical Flicker Fusion}

The measurement of critical-flicker-fusion (CFF) frequency represents one of the most reliable and valid psychophysical indicators of alertness and cortical arousal. CFF is therefore a widely used method for demonstrating drug-induced sedation (Hindmarch, 1982; Smith \& Misiak, 1976). CFF threshold values were recorded by a ZAK Leeds Psychomotor Tester. Twelve CFF measurements were taken at each session, using the psychophysical method of limits, with 6 ascending presentations followed by 6 descending presentations. Mean threshold values were computed on the basis of these 12 runs.

\section{Self-Rating Scales}

The intensity of feelings of activity, alertness, concentration, drowsiness, joyfulness, and relaxation was to be marked on a rating scale ranging from 1 (i.e., not at all) to 6 (i.e., extremely pronounced).

\section{Pharmacological Substances}

AMPT. AMPT blocks the biosynthesis of catecholamines by inhibiting the hydroxylation of tyrosine to L-dopa (DeQuattro \& 
Sjoerdsma, 1968; Sjoerdsma, Engelman, Spector, \& Udenfriend, 1965). Inhibition of this first and rate-limiting step of catecholamine synthesis results in a significant reduction of endogenous levels of catecholamines and their metabolites (Brodie, Murphy, Goodwin, \& Bunney, 1971; Carlsson, Roos, Wålinder, \& Skott. 1973; Hengstmann, Gugler, \& Dengler, 1979). AMPT was administered orally in a total amount of $1,750 \mathrm{mg}$ divided into four doses given $26 \mathrm{~h}(250 \mathrm{mg})$ and 18,9 , and $3 \mathrm{~h}(500 \mathrm{mg})$ before the beginning of the testing session. Lactose in identical capsules was used as a placebo.

Alcohol. Sixty-five min before the testing session, $0.65 \mathrm{~g}$ of $200-$ proof ethanol per kg of body weight was administered, diluted in caffeine-free diet cola with two drops of peppermint oil floated on top of the drink. In the placebo condition, ethanol was omitted. Under each of the experimental conditions, total volume of the drink was $400 \mathrm{ml}$. Saliva specimens were obtained $70 \mathrm{~min}$ after completion of ethanol intake and analyzed immediately after each testing session. For the quantitative enzymatic determination of ethanol intoxication, ethanol in saliva has been measured by means of a narrowbandwidth spectrophotometer (Beckman DU-20) using the Sigma Diagnostics Procedure No. 332-UV. Mean partition ratios for pooled saliva/blood have been shown to be 1.08:1 (Jones, 1979); thus, alcohol in saliva represents a valid indicator of the subjects' blood alcohol concentrations.

\section{HPLC Analysis}

Sample collection. For quantification of DA and its metabolites dihydroxyphenylacetic acid (DOPAC) and homovanillic acid (HVA), a urine specimen was obtained from each subject at the end of the testing session. After the collection, urine samples were adjusted to a $\mathrm{pH}$ of 1.5 with concentrated $\mathrm{HCl}$ and kept at $-70^{\circ} \mathrm{C}$ until tested.

Chromatographic conditions. To analyze urine samples, two piston pumps, Models 302 and 303 with 5 SC pump heads (Gilson Medical Electronics, Middleton, WI), a Rheodyne injection valve with $20-\mu$ l loop, and a $25-\mathrm{cm} C-18$ reverse phase column packed with $5-\mu \mathrm{m}$ particles (Dupont Zorbax, Chadds Ford, PA) were used. In addition, an ESA Coulochem Modell $5100 \mathrm{~A}$ electrochemical detector with guard cell (Model 5020) and high sensitivity analytical cell (Model 5011, ESA Inc., Bedford, MA) were utilized in conjunction with an IBM PC/ 2 computer equipped with the Gilson 714 high-performance liquid chromatography (HPLC) software package (Rainin, Woburn, MA).

Samples were analyzed in a mobile phase consisting of $30 \mathrm{ml}$ of methanol, $0.844 \mathrm{~g}$ of 1 -heptanesulfonic acid, $25.40 \mathrm{~g}$ of citric acid, $15.30 \mathrm{~g}$ of sodium acetate, and $0.113 \mathrm{~g}$ of ethylenediaminetetraacetic acid (EDTA) per liter of HPLC-grade water (Fisher Scientific, NJ). After the mobile phase was filtered through a $0.2-\mu \mathrm{m}$ filter by vacuum suction, it was degassed for 10-15 min before the HPLC system was primed. Recirculation of the mobile phase at a $1-\mathrm{ml} / \mathrm{min}$ flow rate was carried out for approximately 10 days, at which time the mobile phase was replaced.

Reagents. All reagents used, including perchloric acid solution and Tris buffer, were prepared according to Foti, Kimura, DeQuattro, and Lee (1987). Alumina, Super I, Type WA-4: Acid (Sigma Chemical Co., St. Louis, MO) and acid-washed alumina (Bio-Rad, Hercules, CA) were used for the extraction of DA, DOPAC, and HVA. The extraction procedure for urine samples was performed as described by Foti et al. However, a change in this procedure was that $10-\mu \mathrm{l}$ samples were used for injection instead of the $100-\mu \mathrm{l}$ size used by Foti et al. Also, as an internal standard, $50 \mathrm{pM} / \mathrm{ml}$ of DHBA was used.

Analysis. Approximately 10 samples were analyzed each week in a $3 \%$ methanol mobile phase, as described above. The average run time for each sample was $35 \mathrm{~min}$, during which DA and its metabolites were eluted in the order DOPAC, DA, HVA. Standards were prepared from stock solutions $\left(10^{-8} \mathrm{pM} / \mathrm{ml}\right)$ each week and combined to final concentrations of $25 \mathrm{pM} / \mathrm{ml}$ for DOPAC, $50 \mathrm{pM} / \mathrm{ml}$ for $\mathrm{DA}$, and $100 \mathrm{pM} / \mathrm{ml}$ for HVA. Standard peak heights were measured by hand and compared with computer-integrated values using the
Gilson 714 software package to ensure accuracy. Resulting individual urinary DA, DOPAC, and HVA concentrations were standardized by means of respective urinary creatinine levels.

Creatinine determination. Creatinine clearance is frequently used as a standard for the quantification of urinary compounds to become independent of different urinary volumes (Katz, Jenkins, Haley, \& Watson, 1991; Vestergaard \& Leverett, 1958). Creatinine is the final product of creatine catabolism and is excreted at a relatively constant rate. In order to determine the urinary creatinine levels $(\mathrm{mg} / \mathrm{dl})$, we used the Sigma Diagnostics Creatinine Test Kit No. 555. This procedure is based on the colorimetric method originally described by Heinegård and Tiderström (1973). Until they were tested, all urine samples were kept untreated in sealed 1.5-ml Sarstedt containers at $-70^{\circ} \mathrm{C}$, but for no longer than 35 days. The samples were then diluted 15 times and measured in Fisher polystyrene cuvettes in a Beckman DU-20 spectrophotometer at a wavelength of $500 \mathrm{~nm}$. Linearity was present over the entire range of concentrations tested. For standardization, measured DA, DOPAC, and HVA concentrations were divided by their respective creatinine concentrations (catecholamine-to-creatinine ratios).

Time course of the experiment. After a 24 -h pretreatment with AMPT or placebo, ethanol was administered to the subjects at 4:00 p.m., $2 \mathrm{~h}$ after the last AMPT intake; this was followed by a $1-\mathrm{h}$ waiting period. At 5:05 p.m., the testing session started with the choice reaction time task, followed by the self-rating scales. Before the CFF measurement, a saliva sample was obtained. At the end of the 30min testing session, a urine sample was collected.

Data analysis. The combined effects of AMPT and ethanol were evaluated in a single experiment using a completely randomized factorial design. Since there are two treatment factors (AMPT and ethanol), with each treatment having two levels $[A+(=1,750 \mathrm{mg}$ of AMPT $)$ vs. A - (= placebo instead of AMPT); and $E+(=0.65 \mathrm{~g} / \mathrm{kg}$ of ethanol) vs. $E-$ (= placebo instead of ethanol), respectively], the experiment consisted of $2 \times 2=4$ treatment combinations, referred to as $A-/ E-, A-/ E+, A+/ E-$, and $A+/ E+$ conditions. Unlike main-effect means, representing the means across all observations made under a certain treatment [e.g., main-effect mean of $\mathrm{A}+=$ $(\mathrm{A}+/ \mathrm{E}-$ plus $\mathrm{A}+/ \mathrm{E}+) / 2]$, means of each of these treatment combinations are referred to as simple main-effect means (Kirk, 1982) or cell means. All data were analyzed by a two-way analysis of variance (ANOVA), with AMPT and ethanol as the independent treatment factors. Statistically significant interactions were further analyzed by the least significant difference (LSD) test (Kirk, 1982). Since ethanol was only administered to the subjects tested under either the A-/E+ or $\mathrm{A}+/ \mathrm{E}+$ conditions, differences in alcohol concentrations in saliva between these two treatment combinations were evaluated by $t$ test.

\section{RESULTS}

The amount of ethanol administered in the present study resulted in a mean alcohol concentration in saliva of $76.5 \mathrm{mg} / \mathrm{dl}$ and $79.3 \mathrm{mg} / \mathrm{dl}$ for subjects in the $\mathrm{A}-/ \mathrm{E}+$ condition and in the $\mathrm{A}+/ \mathrm{E}+$ condition, respectively. A $t$ test revealed that mean alcohol concentrations did not differ significantly between these experimental conditions $[t(28)=$ 0.43 , n.s.]. In all nonalcohol (E-) subjects, measured alcohol levels were zero. Main-effect means $( \pm S E)$ of the two treatment factors and simple main-effect means $( \pm S E)$ of the four treatment combinations $(\mathrm{A}-/ \mathrm{E}-$, $\mathrm{A}-/ \mathrm{E}+, \mathrm{A}+/ \mathrm{E}-, \mathrm{A}+/ \mathrm{E}+$ ) for all the other dependent variables obtained in the present study are shown in Tables $1 \mathrm{~A}$ and $1 \mathrm{~B}$, respectively.

The main effect of AMPT on dopaminergic activity as indicated by urinary excretion of DA, DOPAC, and HVA was highly significant. Two-way ANOVAs showed a pro- 
nounced AMPT-induced decrease in DA $[F(1,56)=35.42$, $p<.001]$, DOPAC $[F(1,56)=42.73, p<.001]$, and HVA $[F(1,56)=24.61, p<.001]$. However, there were no statistically significant ethanol-induced changes in $\mathrm{DA}[F(1,56)$ $=2.49$, n.s. $]$, DOPAC $[F(1,56)=0.27$, n.s. $]$, or HVA $[F(1,56)=1.66$, n.s. $]$, and there were no interactive effects between AMPT and ethanol on urinary excretion of DA $[F(1,56)=0.05$, n.s. $]$, DOPAC $[F(1,56)=0.07$, n.s. $]$, or HVA $[F(1,56)=0.11$, n.s. $]$. Thus, while ethanol did not seem to affect urinary DA excretion, AMPT proved to be highly effective in reducing dopaminergic activity by inhibition of hydroxylation of tyrosine to L-dopa.

A two-way ANOVA also revealed a significant main effect of ethanol for lift-off time $[F(1,56)=9.46, p<.01]$; mean lift-off time was $382 \mathrm{msec}$ for the $\mathrm{E}-$ group and $413 \mathrm{msec}$ for the $\mathrm{E}+$ group. There was no significant main effect of AMPT $[F(1,56)=0.01$, n.s.]. However, there was a significant interaction between AMPT and ethanol $[F(1,56)=4.03, p<.05]$. As can be seen from Figure 2, lift-off times of the $\mathrm{A}-/ \mathrm{E}+$ and $\mathrm{A}+/ \mathrm{E}+$ conditions were markedly increased as compared with that of the $A-/ E-$ condition ( $p<.001$ and $p<.05$, respectively). In addition, lift-off time for the $\mathrm{A}-/ \mathrm{E}+$ condition was significantly slower than that for the $\mathrm{A}+/ \mathrm{E}-$ condition $(p<.05)$. All other a posteriori pairwise comparisons did not yield statistically significant results. Movement time was not influenced by the drugs administered in the present experiment.

Further, significant main effects of AMPT $[F(1,56)=$ $6.54, p<.05]$, as well as of ethanol $[F(1,56)=24.25, p<$ $.001]$, on CFF thresholds were found. However, there was no significant interaction between AMPT and ethanol $[F(1,56)=.02$, n.s. $]$.

Self-ratings of activity, drowsiness, and relaxation were not significantly affected by either AMPT or ethanol treatment, or by the combination of both. However, the decrease in feelings of activity observed with AMPT just failed to reach statistical significance $[F(1,56)=3.56$, $p=.06]$. While there was no significant main effect of AMPT on self-ratings of alertness, concentration, and joyfulness, ethanol caused a statistically significant decrease in self-ratings of alertness and concentration $[F(1,56)=$ 4.04 and $F(1,56)=4.74$, respectively, both $p s<.05]$ and

Table 1A

Main-Effect Means ( $\pm S E$ ) of the Two Treatment Factors, AMPT and Ethanol

\begin{tabular}{|c|c|c|c|c|c|c|c|c|}
\hline \multirow[b]{4}{*}{ Dependent Variable } & \multicolumn{8}{|c|}{ Treatment Factor } \\
\hline & \multicolumn{4}{|c|}{ AMPT* $^{*}$} & \multicolumn{4}{|c|}{ Ethanol $\dagger$} \\
\hline & \multicolumn{2}{|c|}{$\mathrm{A}-(n=30)$} & \multicolumn{2}{|c|}{$\mathrm{A}+(n=30)$} & \multicolumn{2}{|c|}{$\mathrm{E}-(n=30)$} & \multicolumn{2}{|c|}{$\mathrm{E}+(n=30)$} \\
\hline & $M$ & $S E$ & $M$ & $S E$ & $M$ & $S E$ & $\bar{M}$ & $S E$ \\
\hline Dopamine $(\mathrm{pmol} / \mathrm{ml})$ & 0.685 & 0.056 & 0.299 & 0.034 & 0.443 & 0.053 & 0.542 & 0.063 \\
\hline $\mathrm{DOPAC}(\mathrm{pmol} / \mathrm{ml})$ & 1.998 & 0.189 & 0.579 & 0.101 & 1.233 & 0.208 & 1.345 & 0.192 \\
\hline HVA $(\mathrm{pmol} / \mathrm{ml})$ & 1.231 & 0.149 & 0.443 & 0.054 & 0.735 & 0.127 & 0.939 & 0.138 \\
\hline Lift-off time (msec) & 398 & 8 & 397 & 7 & 382 & 5 & 413 & 9 \\
\hline Movement time (msec) & 190 & 8 & 202 & 8 & 197 & 9 & 196 & 8 \\
\hline $\mathrm{CFF}(\mathrm{Hz})$ & 27.2 & 0.7 & 25.3 & 0.6 & 28.1 & 0.4 & 24.4 & 0.6 \\
\hline \multicolumn{9}{|l|}{ Self-ratings: } \\
\hline activity & 3.8 & 0.2 & 3.3 & 0.2 & 3.5 & 0.2 & 3.6 & 0.2 \\
\hline alertness & 3.8 & 0.2 & 3.6 & 0.2 & 3.9 & 0.2 & 3.4 & 0.2 \\
\hline concentration & 3.3 & 0.2 & 3.4 & 0.2 & 3.6 & 0.2 & 3.1 & 0.2 \\
\hline drowsiness & 3.2 & 0.3 & 3.6 & 0.2 & 3.6 & 0.2 & 3.2 & 0.3 \\
\hline joyfulness & 3.6 & 0.2 & 3.4 & 0.2 & 3.1 & 0.2 & 3.8 & 0.2 \\
\hline relaxation & 4.0 & 0.3 & 4.3 & 0.2 & 4.0 & 0.2 & 4.3 & 0.2 \\
\hline
\end{tabular}

*Factor levels: At $=1,750 \mathrm{mg}$ of AMPT; $\quad$ A- = placebo instead of AMPT; $\quad$ factor levels: $\mathrm{E}+=0.65 \mathrm{~g} / \mathrm{kg}$ of ethanol; $\mathrm{E}-=$ placebo instead of ethanol.

Table 1B

Simple Main-Effect Means $( \pm S E$ ) of the Four Treatment Combinations

\begin{tabular}{|c|c|c|c|c|c|c|c|c|}
\hline \multirow[b]{3}{*}{ Dependent Variable } & \multicolumn{8}{|c|}{ Treatment Combinations } \\
\hline & \multicolumn{2}{|c|}{$\mathrm{A}-/ \mathrm{E}-(n=15)$} & \multicolumn{2}{|c|}{$\mathrm{A}-/ \mathrm{E}+(n=15)$} & \multicolumn{2}{|c|}{$\mathrm{A}+/ \mathrm{E}-(n=15)$} & \multicolumn{2}{|c|}{$\mathrm{A}+/ \mathrm{E}+(n=15)$} \\
\hline & $M$ & $S E$ & $M$ & $S E$ & $M$ & $S E$ & $M$ & $S E$ \\
\hline Dopamine $(\mathrm{pmol} / \mathrm{ml})$ & 0.630 & 0.068 & 0.740 & 0.089 & 0.255 & 0.046 & 0.344 & 0.050 \\
\hline DOPAC $(\mathrm{pmol} / \mathrm{ml})$ & 1.970 & 0.306 & 2.026 & 0.233 & 0.495 & 0.094 & 0.664 & 0.179 \\
\hline HVA (pmol/ml) & 1.155 & 0.196 & 1.308 & 0.230 & 0.315 & 0.056 & 0.571 & 0.081 \\
\hline Lift-off time (msec) & 373 & 6 & 423 & 12 & 392 & 8 & 402 & 13 \\
\hline Movement time (msec) & 186 & 10 & 194 & 13 & 208 & 13 & 197 & 9 \\
\hline $\mathrm{CFF}(\mathrm{Hz})$ & 29.0 & 0.6 & 25.4 & 1.0 & 27.2 & 0.6 & 23.5 & 0.7 \\
\hline \multicolumn{9}{|l|}{ Self-ratings: } \\
\hline activity & 3.8 & 0.3 & 3.8 & 0.3 & 3.3 & 0.2 & 3.3 & 0.3 \\
\hline alertness & 3.8 & 0.3 & 3.7 & 0.3 & 4.1 & 0.2 & 3.1 & 0.2 \\
\hline concentration & 3.7 & 0.3 & 2.9 & 0.2 & 3.5 & 0.2 & 3.2 & 0.2 \\
\hline drowsiness & 3.5 & 0.4 & 2.9 & 0.4 & 3.7 & 0.3 & 3.6 & 0.3 \\
\hline joyfulness & 2.9 & 0.3 & 4.3 & 0.2 & 3.3 & 0.2 & 3.4 & 0.3 \\
\hline relaxation & 3.9 & 0.3 & 4.1 & 0.4 & 4.2 & 0.2 & 4.5 & 0.2 \\
\hline
\end{tabular}




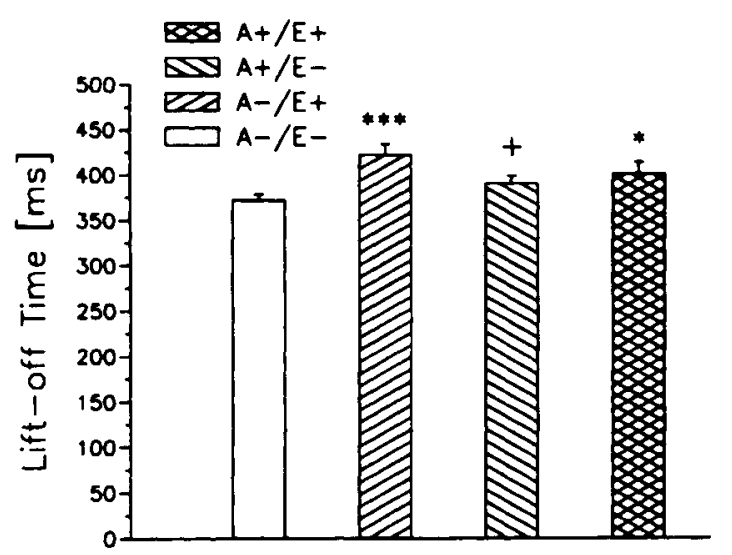

Figure 2. Effects of AMPT and ethanol on lift-off time. *Significantly different from $A-/ E-(p<.05)$. ***Significantly different from $A-/ E-(p<.001)$. + Significantly different from $\mathrm{A}-/ \mathrm{E}+(p<.05)$.

a marked increase in joyfulness $[F(1,56)=6.55, p=$ .01]. A significant interaction between AMPT and ethanol was also found $[F(1,56)=5.37, p<.05]$, indicating that feelings of joyfulness observed under the $A-/ E+$ condition were significantly higher as compared with the $\mathrm{A}-/ \mathrm{E}-(p<.01)$ condition and the $\mathrm{A}+/ \mathrm{E}-$ and $\mathrm{A}+/ \mathrm{E}+$ conditions (both $p s<.05$ ), whereas no differences were found among the latter three conditions (see Figure 3).

\section{DISCUSSION}

In the present study, AMPT did not affect the absorption of alcohol, as indicated by almost identical blood alcohol concentrations of the subjects of the placebo/alcohol and AMPT/alcohol conditions. This finding shows that effects of alcohol that were no longer observed after administration of AMPT are not the consequence of a lower absorption rate and lower blood alcohol levels. Thus, it can be assumed that the inhibition of DA synthesis indeed decreased specific DA-mediated euphoriant effects of alcohol. Therefore, the significantly more pronounced feelings of joyfulness under the placebo/alcohol condition as compared with the AMPT/alcohol condition support the findings of Ahlenius et al. (1973) and Engel and Carlsson (1977). These authors report a significant reduction of euphoric mood induced by alcohol - based on the judgments of three independent, nonintoxicated observers-after pretreatment with 2,000 and $4,000 \mathrm{mg}$ of AMPT in comparison with an untreated alcohol group. In contrast to the present study, no differences were found by self-ratings of the experimental subjects. The reason for this difference can most likely be found in the fact that all the subjects in the above studies were given alcohol and, in addition, they were well informed about the amount of alcohol consumed ( $4 \times 50 \mathrm{ml}$ of Ålborg's Jubileumsaquavit at 10 min intervals), since this study was performed in a social setting. This may have caused the subjects to expect similar euphoric effects of alcohol under both conditions, and thus to deny any differences in the experienced effects.
Such problems were avoided in the present study by the placebo-controlled experimental design. According to our results, pretreatment with AMPT is indeed capable of preventing alcohol-induced euphoria by inhibiting the synthesis of DA and, thus, dopaminergic activity of the mesolimbic DA system.

A further effect caused by alcohol, observed in the present study, was a reduction in feelings of concentration and alertness. These changes were unaffected by treatment with AMPT, suggesting nondopaminergic-mediated actions of alcohol. However, it is conceivable that the reduction in experienced alertness may have been enhanced by the combination of alcohol and AMPT. Ahlenius et al. (1973) and Engel and Carlsson (1977) report a significant, dose-dependent decrease in alertness by the combination of alcohol and AMPT in contrast to alcohol without AMPT. Such an enhancement of sedative effects could not be statistically verified in our study. Since Ahlenius et al. and Engel and Carlsson used doses of AMPT of 2,000 and $4,000 \mathrm{mg}$, it is possible that such effects only occur after doses of more than $1,750 \mathrm{mg}$, the dose used in our experiment. The relative lack of sedative effects experienced by our subjects is also reflected by the observation that drowsiness was not significantly affected by alcohol, AMPT, or the combination of both substances.

Unlike self-ratings of alertness and drowsiness, CFF can be assumed to be an objective indicator for pharmacologically induced alertness or CNS activation. Here again, a significant decrease was observed not only under the influence of alcohol but also, although somewhat less, under AMPT, indicating a reduction of central nervous arousal. However, no potentiation was seen by the combination of alcohol and AMPT on this indicator of central activity. In general, the results obtained with subjective and objective measures of alertness suggest a decrease in central activation under alcohol that is manifested much more clearly through the objective parameter CFF than it is through the ratings of subjective feelings. An explanation for the higher sensitivity of objective measures of central activation in

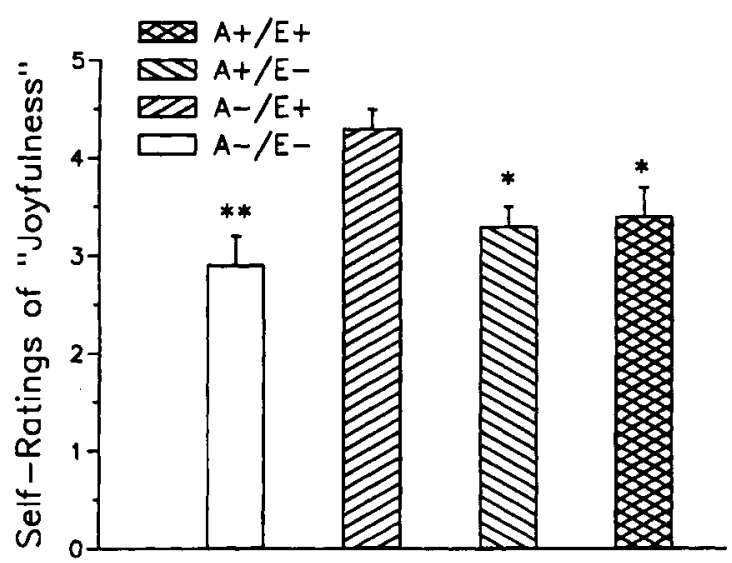

Figure 3. Effects of AMPT and ethanol on feelings of joyfulness. *Significantly different from $A-/ E+(p<.05)$. **Significantly different from $A-/ E+(p<.01)$. 
conjunction with alcohol-induced effects could perhaps be found in the fact that the experienced euphoric action of alcohol antagonizes, at least partially, the subjective experience of sedative effects.

The effects of alcohol on the brain vary as a function of dose. In general, small amounts of alcohol result in stimulating effects whereas increased concentrations cause sedative effects. This biphasic action of alcohol can be explained through differential sensitivity of catecholaminergic, in particular dopaminergic, and GABAergic mechanisms in response to alcohol (Engel \& Liljequist, 1983). According to this model, euphoric actions of small doses of alcohol are mediated by dopaminergic mechanisms and sedative actions of larger doses of alcohol are mediated by GABAergic mechanisms. Small doses of alcohol produce a more pronounced effect on the DA system than on the GABA system, masking the sedative effect of GABAergic activation and, thus, causing stimulating and euphoric actions. Only at higher doses of alcohol do the effects on the GABAergic system predominate. Since in the present study alcohol caused a significant decrease in experienced alertness and concentration as well as reduced performance on CFF, it can be assumed that these sedative effects are due to actions on the GABA system. One may wonder how blood alcohol concentrations as low as approximately $78 \mathrm{mg} / \mathrm{dL}$ can already cause these sedative effects. However, if one considers that the subjects of the present study were either nondrinkers or extremely moderate drinkers who generally consumed very little alcohol, it is possible that these sedative effects were caused by the actions of alcohol $(0.65 \mathrm{~g} / \mathrm{kg})$ on an unprepared organism not used to alcohol. There was no chance for tolerance to the effects of alcohol to develop, resulting in high sensitivity to alcohol effects.

GABAergic mechanisms do not only play a major role in central deactivation processes, they may also be involved in the mesostriatal regulation of behavioral responsiveness. Since the mesostriatal DA neurons interact with the GABA neurons representing the hypothetical inhibitory control mechanism, it is important to know whether the changes in choice reaction time found in the present study primarily reflect dopaminergic or GABAergic effects. Since movement time, representing the primary motor component of choice-reaction time, was insensitive to AMPT and alcohol, we can only use the results of lift-off time to answer this question. Certainly, lift-off time comprises part of a motor component. But because the motor response to the onset of the stimulus light is almost a reflex (Frewer \& Hindmarch, 1988), it can safely be assumed that lift-off time "represents the latency of processes other than motor execution and thus reflects aspects of the speed of stimulus recognition and response organization" (Frewer \& Hindmarch, 1988, p. 107). Obviously, these processes correspond to the functional steps necessary for eliciting appropriate behavioral responses as proposed by the model describing the action of the mesostriatal DA system. The main effect of alcohol indicating marked decreases in feelings of alertness and concentration as well as a pronounced increase in lift-off time may be indicative of a GABAergic deteriorating effect. This interpretation is supported by the significant interaction between AMPT and alcohol found for lift-off time in the following way: Although liftoff times of the placebo/alcohol and AMPT/alcohol conditions were significantly increased as compared with the placebo/placebo condition, lift-off time for the AMPT/ placebo condition was significantly faster than that for the placebo/alcohol condition. This pattern of results points to the conclusion that the impairing effect of alcohol is primarily mediated by GABAergic mechanisms. At the same time, AMPT does not appear to effectively decrease dopaminergic activity of the mesostriatal system, since, if AMPT affected activity of mesostriatal DA neurons, a potentiating effect of AMPT and alcohol should have become evident. In this respect, the results of the present study show that, unlike functions mediated by the mesolimbic DA system (e.g., euphoric actions of alcohol), functions mediated by the mesostriatal DA system are less likely to be affected by an AMPT-induced reduction of dopaminergic activity. This conclusion may be surprising and unexpected, particularly if one considers that the pharmacologically induced decrease of activity of the mesolimbic DA system by AMPT was highly effective in reducing alcohol-mediated euphoria in the present experiment. However, there is converging neurobiological evidence for such an interpretation. According to Andén et al. (1984), there exists a welldescribed pathway from the striatum to the substantia nigra, most probably providing negative neuronal feedback. No similar feedback loop from the nucleus accumbens to the VTA seems to exist (Andén et al., 1984; Nauta, Smith, Fall, \& Domesick, 1978). The absence of such transneuronal feedback may cause those functions mediated by the dopaminergic pathway from the VTA to the nucleus accumbens (i.e., the mesolimbic DA system) to be particularly susceptible to any pharmacologically induced changes in dopaminergic activity. This may explain why pretreatment with AMPT effectively blocked alcoholinduced euphoria, which is mediated by the mesolimbic DA system, whereas no additive effect of AMPT and alcohol could be shown for lift-off time as an indicator of dopaminergic activity in the mesostriatal pathway. In this view, the pattern of results that showed more pronounced effects of the DA antagonist AMPT on the mesolimbic as compared with the mesostriatal DA system is consistent with the neurophysiological properties of these pathways. The relatively high capacity of the mesostriatal DA system for compensation has already been demonstrated in a human study (Rammsayer et al., 1993). On the basis of these results, it is not surprising that lift-off time was not affected by AMPT pretreatment. Further, this suggestson the basis of the Stricker and Zigmond (1986) modelthat the observed changes in lift-off time are caused by alcohol-induced GABAergic effects.

The analysis of the interaction between AMPT and alcohol lends additional support for such an interpretation. Whereas lift-off times are not different between the placebo/ placebo and the AMPT/placebo conditions, lift-off time is considerably slower under the placebo/alcohol condition. Although lift-off time under the influence of AMPT/alco- 
hol is higher than that under placebo/placebo, it is reduced as compared with placebo/alcohol, but not to a significant level. Apparently, alcohol causes an increase in the activity of the GABAergic inhibitory control mechanism and, thus, impairment in processing of sensory input, which is manifested through a deceleration of lift-off time under the placebo/alcohol condition, as well as under the AMPT/ alcohol condition. On the other hand, no evidence could be found for inhibition of the mesostriatal DA system, since the AMPT/placebo group was not different from the placebo/placebo group. Of importance for this conclusion is the fact that there was a lack of potentiation of the pharmacological effects by the combination of AMPT and alcohol. If AMPT decreased the activity of the mesostriatal DA system and, at the same time, an additional dose of alcohol increased the GABAergic activity of the inhibitory control mechanism, a more severe deteriorating effect on lift-off time would have been expected in comparison with the one observed under the placebo/alcohol condition. However, our results clearly do not support any conclusion suggesting a potentiating effect of AMPT and alcohol.

Finally, it is important to note that the biochemical results are in line with the interpretations drawn from the behavioral results. The HPLC analysis clearly demonstrated the inhibitory action of AMPT on DA synthesis, as indicated by a pronounced decrease in urinary excretion of DA and its metabolites DOPAC and HVA. Pretreatment with $1,750 \mathrm{mg}$ of AMPT caused a reduction in the urinary DA excretion of $56.5 \%$ and a corresponding decrease of $71.0 \%$ and $64.2 \%$ in excretion of the metabolites DOPAC and HVA, respectively. These results of the HPLC analysis are in agreement with the AMPT-induced reductions of catecholamine synthesis in the range of $40 \%-80 \%$ as reported by Engelman, Horwitz, Jequier, and Sjoerdsma (1968) and Hengstmann et al. (1979). The major source of free urinary DA appears to originate from the decarboxylation of circulating L-dopa. It does not make a large contribution to the total amount of metabolites and is not representative of dopaminergic activity in the brain exclusively (Kopin, 1985). Nevertheless, reduction of urinary DA levels can be used as an indicator that indirectly represents the antagonistic effects of AMPT on brain DA activity. On the other hand, DOPAC and, especially, HVA, the major urinary metabolite of DA, can be considered to more directly represent central dopaminergic activity (Kopin, 1985). Despite the fact that conclusions of this study are based on urinary excretion of DA and its metabolites, the action of AMPT clearly involves the central nervous system. On the basis of animal models and the potentiation of the antipsychotic effects of neuroleptic treatment in chronic schizophrenics, Carlsson et al. (1973) concluded that AMPT in doses of approximately $2,000 \mathrm{mg}$ per day markedly inhibits synthesis of catecholamines in the brain. In addition, these authors found that AMPT caused a $70 \%-90 \%$ decrease in cerebrospinal-fluid HVA levels in five out of six subjects. Compared with the $64.2 \%$ decrease in urinary HVA levels obtained with $1,750 \mathrm{mg}$ per day in the present study, the inhibiting effect of AMPT on brain DA seems even more pronounced if measured in cerebrospinal fluid. Further evidence for an AMPT-induced blockade of central dopaminergic activity is provided by clinical studies of manic and schizophrenic patients (e.g., Brodie et al., 1971; Magelund, Gerlach, \& Casey, 1979) and animal studies (e.g., Ahlenius \& Engel, 1973; Davis \& Smith, 1973). Therefore, we can safely assume that in the present experiment, the effective level of central dopaminergic activity had been dramatically decreased by AMPT. On the other hand, dopaminergic activity as indicated by urinary excretion of DA, DOPAC, and HVA proved to be virtually unaffected by the moderate dose of alcohol administered in this study.

In conclusion, the outcome of the present study showed that pretreatment with a specific inhibitor of tyrosine hydroxylase successfully prevents the euphoric action of alcohol but not the decrease in behavioral responsiveness. This finding provides additional evidence for the significance of brain DA in mediating alcohol-induced euphoria. Our laboratory data suggest that AMPT may have potential value in treatment of alcohol abuse. According to the notion put forward by Davis and Smith (1973), the benefit of AMPT treatment may be twofold. First, alcohol consumption maintained by the euphoric action of alcohol, representing primary reinforcement, should be extinguished, and, moreover, secondary reinforcers, such as environmental stimuli associated with alcohol abuse, should also lose their effectiveness due to lack of reinforcement. However, more clinical trials are necessary to evaluate the effectiveness of AMPT in treatment of alcohol abuse. Further, the pattern of results observed in the present study points to the crucial role of the functional interaction between dopaminergic and GABAergic mechanisms for understanding the behavioral effects of alcohol. Finally, it can be stated that neurobiological models of behavioral regulation developed in animals can be successfully applied to human studies and, thus, represent an important avenue in understanding the psychobiological basis of human behavior.

\section{REFERENCES}

Ahlenius, S., Carlsson, A., Engel, J., Svensson, T., \& SöderSTEN, P. (1973). Antagonism by alpha-methyltyrosine of the ethanolinduced stimulation and euphoria in man. Clinical Pharmacology \& Therapeutics, 14, 586-591.

AhleniUs, S., \& ENGEL, J. (1973). On the interaction between pimozide and $\alpha$-methyltyrosine. Journal of Pharmacy \& Pharmacology, 25, 172-174.

Andén, N.-E., Alander, T., Grabowska-Andén, M., Liluenberg, B., LindGREN, S., \& THORNSTRÖM, U. (1984). The pharmacology of preand postsynaptic dopamine receptors: Differential effects of dopamine receptor agonists and antagonists. In Catecholamines: Neuropharmacology and central nervous system. Theoretical aspects (pp. 19-24). New York: Alan R. Liss.

Björklund, A., \& Lindvall, O. (1986). Catecholaminergic brain stem regulatory systems. In Handbook of physiology: Section 1. The nervous system (Vol. 4, pp. 155-235). Bethesda, MD: American Physiological Society.

Bozarth, M. A. (1987). Ventral tegmental reward system. In J. Engel, L. Oreland, D. H. Ingvar, B. Pernow, S. Rössner, \& L. A. Pellborn (Eds.), Brain reward systems and abuse (pp. 1-33). New York: Raven Press. Bozarth, M. A., \& Wise, R. A. (1981). Heroin reward is dependent on a dopaminergic substrate. Life Sciences, 29, 1881-1886.

Brodie, H. K. H., Murphy, D. L., Goodwin, F. K., \& Bunney, W. E. 
(1971). Catecholamines and mania: The effect of $\alpha$-methyl-paratyrosine on manic behavior and catecholamine metabolism. Clinical Pharmacology \& Therapeutics, 12, 218-224.

Carlsson, A., Roos, B.-E., Wålinder, J., \& Skott, A. (1973). Further studies on the mechanism of antipsychotic action: Potentiation by $\alpha$-Methyltyrosine of thioridazine effects in chronic schizophrenics. Journal of Neural Transmission, 34, 125-132.

DaVIS, W. M., \& SMITH, S. G. (1973). Blocking effects of $\alpha$-methyltyrosine on amphetamine based reinforcement. Journal of Pharmacy \& Pharmacology, 25, 174-177.

DeQuattro, V., \& SJoerdsma, A. (1968). Catecholamine turnover in normotensive and hypertensive man: Effects of antiadrenergic drugs. Journal of Clinical Investigation, 47, 2359-2373.

DiAL, J. (1992). The interaction of alcohol and cocaine: A review. Psychobiology, 20, 179-184.

ENGEL, J., \& CARLSSON, A. (1977). Catecholamines in behavior. In W. S. Essman \& L. Valzelli (Eds.), Current developments in psychopharmacology (Vol. 4, pp. 1-32). New York: Spectrum.

ENGEL, J., \& LILJEQUIST, S. (1983). The involvement of different central neurotransmitters in mediating stimulatory and sedative effects of ethanol. In L. A. Pohorecky \& J. Brick (Eds.), Stress and alcohol use (pp. 153-169). New York: Elsevier.

ENGEL, J., Strömbom, U., Svensson, T. H., \& Waldeck, B. (1974). Suppression by $\alpha$-methyltyrosine of ethanol-induced locomotor stimulation: Partial reversal by L-dopa. Psychopharmacologia, 37, 275279.

Engelman, K., Horwitz, D., Jequier, E., \& Sjoerdsma, A. (1968). Biochemical and pharmacologic effects of $\alpha$-methyltyrosine in man. Journal of Clinical Investigation, 47, 577-594.

Fibiger, H. C., \& Phillips, A. G. (1986). Reward, motivation, cognition: Psychobiology of mesotelencephalic dopamine systems. In Handbook of physiology: Section 1. The nervous system (Vol. 4, pp. 647-675). Bethesda, MD: American Physiological Society.

Fibiger, H. C., \& PHILlips, A. G. (1987). Role of catecholamine transmitters in brain reward systems: Implications for the neurobiology of affect. In J. Engel, L. Oreland, D. H. Ingvar, B. Pernow, S. Rössner, \& L. A. Pellborn (Eds.), Brain reward systems and abuse (pp. 61-74). New York: Raven Press.

Foti, A., Kimura, S., DeQuattro, V., \& LeE, D. (1987). Liquidchromatographic measurement of catecholamines and metabolites in plasma and urine. Clinical Chemistry, 33, 2209-2213.

Frewer, L. J., \& HiNDMARCH, I. (1988). The effect of time of day, age, and anxiety on a choice reaction task. In I. Hindmarch, B. Aufdembrinke, \& $\mathrm{H}$. Ott (Eds.), Psychopharmacology and reaction time (pp. 103-114). Chichester, U.K.: Wiley.

Gallistel, C. R., Shizgal, P., \& Yeomans, J. (1981). A portrait of the substrate for self-stimulation. Psychological Review, 88, 228-273.

Heffner, T. G., Zigmond, M. J., \& Stricker, E. M. (1977). Effects of dopaminergic agonists and antagonists on feeding in intact and 6hydroxydopamine-treated rats. Journal of Pharmacology \& Experimental Therapeutics, 14, 380-399.

HEINEgÅRD, D., \& TiderSTRöM, G. (1973). Determination of serum creatinine by a direct colorimetric method. Clinica Chimica Acta, $\mathbf{4 3}$, 305-310.

Hengstmann, J. H., Gugler, R., \& DengleR, H. J. (1979). Malignant pheochromocytoma: Effect of oral $\alpha$-methyl-p-tyrosine upon catecholamine metabolism. Klinische Wochenschrift, 57, 351-355.

HindMARCH, I. (1982). Critical flicker fusion frequency (CFF): The effects of psychotropic compounds. Pharmacopsychiatry, 15 (Suppl. 1), 44-48.

JONES, A. W. (1979). Distribution of ethanol between saliva and blood in man. Clinical \& Experimental Pharmacology \& Physiology, 6, 53-59.
JöNSSON, L.-E., ÄNGGÅRD, E., \& GUNNE, L.-M. (1971). Blockade of intravenous amphetamine euphoria in man. Clinical Pharmacology \& Therapeutics, 12, 889-896.

JÖNSSON, L.-E., GUNNE, L.-M., \& ÄNGGÅRD, E. (1969). Effects of alpha-methyltyrosine in amphetamine-dependent subjects. Pharmacologia Clinica, 2, 27-29.

Katz, V. L., Jenkins, T., Haley, L., \& Watson, A. B. (1991). Catecholamine levels in pregnant physicians and nurses: A pilot study of stress and pregnancy. Obstetrics \& Gynecology, 77, 338-342.

KhatiB, S. A., MurPhy, J. M., \& McBride, W. J. (1988). Biochemical evidence for activation of specific monoamine pathways by ethanol. Alcohol, 5, 295-299.

KIRK, R. E. (1982). Experimental design: Procedures for the behavioral sciences. Belmont, CA: Wadsworth.

KoPIN, I. J. (1985). Catecholamine metabolism: Basic aspects and clinical significance. Pharmacological Reviews, 37, 333-364.

LEE, N. M., \& BeCKER, C. E. (1989). The alcohols. In B. G. Katzung (Ed.), Basic and clinical pharmacology (pp. 278-286). Norwalk, CT: Appleton \& Lange.

Magelund, G., Gerlach, J., \& Casey, D. E. (1979). Neurolepticpotentiating effect of $\alpha$-methyl-p-tyrosine compared with haloperidol and placebo in a double-blind cross-over trial. Acta Psychiatrica Scandinavica, 60, 185-189.

Nauta, W. J. H., Smith, G. P., Fall, R. L. M., \& Domesick, V. B. (1978). Efferent connection and nigral afferents of the nucleus accumbens septi in the rat. Neuroscience, 3, 385-401.

NETTER, P., \& VOGEL, W. H. (1995). Questionnaire on alcohol consumption $(Q A C)$. Unpublished manuscript. Giessen, Germany: University of Giessen, Department of Psychology.

Rammsayer, T., Netter, P., \& Vogel, W. H. (1993). A neurochemical model underlying differences in reaction times between introverts and extroverts. Personality \& Individual Differences, 14, 701-712.

Sjoerdsma, A., Engelman, K., Spector, S., \& Udenfriend, S. (1965). Inhibition of catecholamine synthesis in man with alpha-methyl-tyrosine, an inhibitor of tyrosine hydroxylase. Lancet, 2, 1092-1094.

SMITH, J. M., \& MisiaK, H. (1976). Critical flicker frequency (CFF) and psychotropic drugs in normal human subjects: A review. Psychopharmacology, 47, 175-182.

SPIELberger, C. D., GorsuCH, R. L., \& LUSHENE, R. E. (1970). Manual for the state-trait anxiety inventory. Palo Alto, CA: Consulting Psychologists Press.

Stricker, E. M., \& Zigmond, M. J. (1986). Brain monoamines, homeostasis, and adaptive behavior. In Handbook of physiology: Section 1. The nervous system (Vol. 4, pp. 677-700). Bethesda, MD: American Physiological Society.

VeSTERGAARD, P., \& LEVERETT, R. (1958). Constancy of urinary creatinine excretion. Journal of Laboratory \& Clinical Medicine, 51, $211-218$.

WISE, R. A. (1983). Brain neuronal systems mediating reward processes. In J. E. Smith \& J. D. Lane (Eds.), Neurobiology of opiate reward processes (pp. 405-437). Amsterdam: Elsevier.

WISE, R. A. (1988). The neurobiology of craving: Implications for the understanding and treatment of addiction. Journal of Abnormal Psychology, 97, 118-132.

Zigmond, M. J., Heffner, T. G., \& Stricker, E. M. (1980). The effect of altered dopaminergic activity on food intake in the rat: Evidence for an optimal level of dopaminergic activity for behavior. Progress in Neuro-Psychopharmacology, 4, 351-362.

(Manuscript received June 27, 1994; revision accepted for publication April 19, 1995.) 